\title{
Cytochrome 4ZI Expression is Associated with Unfavorable Survival in Triple-Negative Breast Cancers
}

Yousef $M$ Al-saraireh (D) Fatemah OFO Alshammari $^{2}$ Ahmed MM Youssef iD $^{3}$

Fatima Al-Tarawneh ${ }^{4}$

Sameeh Al-Sarayreh ${ }^{5}$

Ghadeer H Almuhaisen ${ }^{6}$

Anas O Satari ${ }^{7}$

Jehad Al-Shuneigat ${ }^{5}$

Hamzeh Mohammad Alrawashdeh ${ }^{8}$

'Department of Pharmacology, Faculty of Medicine, Mutah University, Al-Karak, Jordan; ${ }^{2}$ Department of Medical Laboratory Technology, Faculty of Health Sciences, The Public Authority for Applied Education and Training, Shuwaikh, Kuwait; ${ }^{3}$ Department of Pharmacology, Faculty of Pharmacy, Mutah University, Al-Karak, Jordan; ${ }^{4}$ Department of Allied Medical Sciences, Faculty of Al-Karak, Al-Balqa Applied University, Al-Karak, Jordan; ${ }^{5}$ Department of Biochemistry and Molecular Biology, Faculty of Medicine, Mutah University, Al-Karak, Jordan; ${ }^{6}$ Department of Microbiology and Pathology, Faculty of Medicine, Mutah University, Al-Karak, Jordan; ${ }^{7}$ Faculty of Medicine, Mutah University, Al-Karak, Jordan; ${ }^{8}$ Department of Ophthalmology, Sharif Eye Centers, Irbid, Jordan
Correspondence: Yousef M Al-saraireh Department of Pharmacology, Faculty of Medicine, Mutah University, P.O. Box: 7, Al-Karak, 61710, Jordan

Tel +962 799172658

Email Yousef.sar@mutah.edu.jo
Purpose: Triple-negative breast cancer (TNBC) is characterized by high mortality rate, and its clinical management is difficult and complex. Therefore, there is a need for extensive efforts aimed at accelerating the discovery of novel therapies for TNBC. CYP4Z1 has been implicated in the development of breast cancer. The current study aimed at characterizing the expression of CYP4Z1 on TNBC.

Materials and Methods: Using immunohistochemistry, CYP4Z1 expression was evaluated on 122 TNBC samples, four samples of breast cancers expressing ER, PR, and HER-2, and four samples of normal breast tissues. The association between the enzyme and various histopathological features and survival of patients were determined.

Results: CYP4Z1 was strongly expressed in $83.3 \%$ of various histopathological subtypes of TNBC, when compared to negative expression in normal breast tissues. Interestingly, there were marked variations in CYP4Z1 expression with respect to histopathology subtype, histological grade, histological stage and tumor diameter. There was a high incidence of CYP4Z1 expression in patients with advanced grades, late stages and larger tumor sizes. Importantly, CYP4Z1 expression was correlated with the survival of TNBC patients, but it was an independent determinant of the poor prognosis of TNBC $(\mathrm{p}<0.05)$.

Conclusion: CYP4ZI may be a potential biomarker or target for evolving new CYP4Z1targeted treatments.

Keywords: triple-negative breast cancer, cytochrome P450, immunohistochemistry

\section{Introduction}

TNBC is a heterogeneous form of mammary cancer. It lacks the most prevalent biological markers of breast cancer, including the receptors for progesterone (PR), estrogen (ER), and human epidermal growth factor 2 (HER2). ${ }^{1}$ Approximately 15 $20 \%$ of breast carcinoma, and $25 \%$ of mortalities associated with breast cancer are due to TNBC. The relative survival for $\mathrm{TNBC}$ is $77 \%$, relative to $93 \%$ for other subtypes of breast cancers. ${ }^{2}$ Thus, in terms of aggressiveness, TNBC is the most potent breast cancer subtype, and it is known for early recurrence, metastatic dissemination and poor prognosis. Since most of the traditional therapies for breast cancer rely on the expressions of ER, PR and HER2, the treatment of TNBC is difficult and challenging. ${ }^{1}$ Therefore, there is a need for identification of new biomarkers or drug targets for improving treatment outcomes in TNBC patients.

Expressions of orphan cytochrome P450s (CYPs) in cancers are of particular interest in diagnosis and targeted therapy. One of the CYP enzymes that is of 
particular interest to researchers due to its aberrant expression in cancers is CYP4Z1. Its expression has been extensively analyzed in tumor cell lines. However, not many of these analyses have been confirmed in clinical samples. Studies have shown that CYP4Z1 is selectively expressed in mammary tissues, relative to almost negative expression in other healthy tissues. ${ }^{3}$ It is of importance that studies have reported uniquely strong expression of CYP4Z1 in several cancers. ${ }^{4-9}$ Its expression has been linked with poor tumor grade and higher patient mortality, and it has been proposed as a biomarker for ovarian, prostate and cervical cancers. ${ }^{5,6,10,11}$ Interestingly, an abnormal translocation of CYP4Z1 expression on the surface of breast cancer cells has been reported, but no expression was observed in healthy control mammary cells. ${ }^{12}$ This pattern of aberrant expression promotes the generation of CYP4Z1 auto-antibodies in sera of breast cancer patients, when compared to healthy controls, and it may function as a clinical biomarker for breast cancer. ${ }^{13}$ However, there is a need for further studies on whether CYP4Z1 autoantibodies are produced in cancers expressing CYP4Z1.

Several studies have attempted to investigate the biochemical and underlying mechanisms of CYP4Z1 in tumor malignancy. Yu et al studied the role of CYP4Z1 in tumor malignancy using in vitro and in vivo models. It was found that CYP4Z1 expression significantly increased tumor proliferation, tumor vascularization and migration of cancer cells. Moreover, CYP4Z1 overexpression enhanced the expression of the pro-angiogenic factor, ie, vascular endothelial growth factor A (VEGF-A), and reduced the expression of tumor suppressor tissue inhibitor of metalloproteinases 2 (TIMP-2) in cancer cells, relative to control cells. Moreover, there were reductions in concentrations of fatty acids (myristate and laurate), and increased levels of 20-HETE. ${ }^{10}$ Earlier studies have reported a tendency to hydroxylation, where CYP4Z1 metabolized myristate and laurate to $\omega$-hydroxylated derivatives, and arachidonate to 20-hydroxyeicosatetraenoic acid (20-HETE). ${ }^{10,14}$ In contrast, a recent study identified an epoxygenase catalytic activity of CYP4Z1, which converts arachidonate to 14,15-epoxyeicosatrienoate $\left(14,15\right.$-EET). ${ }^{15}$ The 14,15 EET is a ligand that promotes tumor growth and angiogenesis. ${ }^{16,17}$ Further biochemical investigations are needed on the role of CYP4Z1 in the generation of these metabolites, as well as their biological effects on tumor progression.

As the current treatment approaches for TNBC are limited and complex, novel treatment strategies, particularly for recurrent and metastatic diseases, are urgently required. Selective mammary expression of CYP4Z1 provides a new opportunity to explore for treatment of breast cancer, particularly TNBC, because CYP inhibitors developed for anticancer treatment have already proved successful like aromatase (CYP19) inhibitors. ${ }^{18}$ Recently, in a large group of breast cancers, the expression of CYP4Z1 expression and its association with various indexes of prognosis such as AR, EGFR, ER, PR, HER-2 and P53, were demonstrated. ${ }^{8}$ Since there are no studies examined CYP4Z1expression in TNBC, this research was carried out to investigate the expression of CYP4Z1 in TNBC, and its association with prognosis.

\section{Materials and Methodology Tissue Specimens}

Before the start of this investigation, an ethical approval was obtained from the Institutional Review and Ethics Committee, Faculty of Medicine, Mutah University (Reference No.6012021 date: 20/01/2021). Informed patient consent was waived due to an exemption for the use of archived paraffin cervical tissue samples and for review patients' records granted by the Institutional Review and Ethics Committee, Faculty of Medicine, Mutah University. Moreover, patients' data were anonymized and maintained with confidentiality. The study protocol was in line with the guidelines of Helsinki Declaration. Samples of breast cancer, including TNBC and normal breast tissues, were submitted to the Department of Pathology of the King Hussein Medical Hospital, Amman, and King Abdullah University Hospital, Jordan during the seven-year period (20132020). All specimens were formalin-fixed, followed by processing and embedding in paraffin wax. The samples were sectioned into 5- $\mu \mathrm{m}$ slices, which were subjected to staining with $\mathrm{H} \& \mathrm{E}$ for routine light microscopy. The inclusion criteria for this study were female patients diagnosed with TNBC aged 18-70 years (122 cases). Moreover, four samples of breast cancers expressing ER, PR, and HER-2 hormone receptors were also included in the study to compare CYP4Z1 expression between TNBC and positive hormone receptors breast cancer samples. The study also consisted of four normal breast samples as control group. The exclusion criterion was that patients had chemotherapy or radiotherapy prior to surgery. The panel of breast tumors consisted of 103 invasive 
carcinomas of no special type, eight medullary carcinomas, three invasive lobular carcinomas, three mixed ductal and lobular carcinomas, two mucinous adenocarcinomas, two invasive micropapillary carcinomas, two neuroendocrine carcinomas, two carcinoids and one apocrine carcinoma. Patients' data on tumor histology, clinical stage, tumor grade, age and expression levels of biomarkers ER, PR, and HER-2 were obtained from their medical records. Since invasive carcinoma of no special type was the main pathologic type in this study (103 cases), survival data for these individuals were obtained from follow-up records ranging from 6 to 60 months (median, 42 months). Overall survival was measured from the surgery date to the death date or the final follow-up appointment.

\section{Immunohistochemistry}

The tissue slices were subjected to dewaxing and rehydration using routine protocols (gradient concentrations of alcohol, and finally in distilled water). Following the inhibition of endogenous peroxidase with $3 \% \mathrm{H}_{2} \mathrm{O}_{2}$, the tissues were antigen-retrieved via microwaving in buffered medium. Washing was followed by incubation of the slides overnight with CYP4Z1 polyclonal antibody $(5 \mu \mathrm{g} / \mathrm{mL}$, NBP1-91817, Novus biological, USA) at $4^{\circ} \mathrm{C}$. After washing, the sections were treated with ImmPRESS (Peroxidase) polymer goat anti-rabbit IgG for half an hour at laboratory temperature. After incubating the slides with the chromogen DAB, they were finally exposed to Harris's hematoxylin and subjected to dehydration, followed by mounting with coverslips. Breast cancer tissue was used as positive control. For negative control (procedural control), the primary antibody was substituted with normal goat serum. The antibody affinity for CYP4Z1 was confirmed with Western blotting using whole CYP4Z1 isogenic cell lysates (results not displayed). Moreover, the specificity of the primary antibody was further confirmed by blocking CYP4Z1 antibody with CYP4Z1 blocking protein (H00199974-P01, Novus Biological, USA). In this case, CYP4Z1primary antibody was pre-incubated with CYP4Z1 blocking peptide for 1 hour at room temperature prior to application to tissue specimens. The staining density was determined and compared between tissues incubated with CYP4Z1 antibody and tissues treated with blocked antibody. The resultant slides were assessed with a Leica DMRB microscope equipped with a JVC video camera. The images were digitally collected and subjected to processing.

\section{Scoring of CYP4ZI Expression}

The expression level of CYP4Z1 was scored by two independent pathologists using Allred scoring system. ${ }^{19-22}$ This system combines the percentage of membranous or cytoplasmic-stained tumor cells (CYP4Z1-positive staining) and the intensity of the staining. The percentage of CYP4Z1 positively stained cells was rated on a score scale of $0-5$. A score of 0 (negative) corresponded to a lack of expression. Sections which showed staining of less than $1 \%$ were scored 1 . A score of 2 indicated positive staining in $1-10 \%$ of the cells. Sections which showed staining between $11 \%$ and $33 \%$ were scored 3 . A score of 4 indicated staining between $34 \%$ and $66 \%$ of the cells. Finally, a score of 5 corresponded to positive staining in excess of $67 \%$ of the cells. Staining intensity was scored on a scale of $0-3$ viz: $0,1,2$ and 3 for ve, weak, moderate and strong staining, respectively. The final score was obtained as the sum of percentage score and intensity score, thereby generating eight possible numbers of scores. A result score of less than 2 indicated negative CYP4Z1 expression, while scores between 3-8 indicated positive CYP4Z1 expression.

\section{Statistics}

Data analysis was carried out using SPSS 19. Results are presented as frequency and percentage. A one-way analysis of variance and Chi-square test were applied to measure differences amongst variables. The overall survival of patients was evaluated using Kaplan-Meier procedure. Statistical significance was determined using Log rank test. The prognostic value of CYP4Z1 was determined using univariate and multivariate Cox regression. Differences were deemed significant at $p<0.05$.

\section{Results}

\section{Baseline Demography and Clinicopathology of Patients}

Table 1 shows that a total of 122 females with TNBC, four females with breast cancers expressing ER, PR, and HER-2 hormone receptors and four females with normal pathology (mean age $=51.4 \pm 12.2$ years) were enrolled in this study. Sixty-nine (69) patients (53.1\%) were under the age of 50 years, while 61 patients $(46.9 \%)$ were aged more than 50 years. Most of the patients were at tumor stage II (92 cases, $73 \%$ ), while 32 patients $(25.4 \%)$ and two patients (1.6\%) were at tumor stage III and tumor stage I, respectively. There were 63 patients (50\%) with grade II, and 63 patients $(47.6 \%)$ with grade III. The other three patients $(2.4 \%)$ were with 
Table I Baseline Demographic and Clinicopathologic Features of TNBC Patients

\begin{tabular}{|c|c|c|c|}
\hline \multirow{2}{*}{ Characteristic } & \multicolumn{2}{|c|}{ CYP4ZI Expression } & \multirow[t]{2}{*}{$P$ value } \\
\hline & Negative $n=25(16.7 \%)$ & Positive $n=105(83.3 \%)$ & \\
\hline \multicolumn{4}{|l|}{ Age: } \\
\hline$<50(n=69,53.1 \%)$ & $16(23.2 \%)$ & $53(76.8 \%)$ & 0.160 \\
\hline$\geq 50(n=61,46.9 \%)$ & $9(14.8 \%)$ & $52(85.2 \%)$ & \\
\hline \multicolumn{4}{|l|}{ Pathology subtype: } \\
\hline Invasive carcinoma of no special type $(n=103,79.2 \%)$ & 18 (I7.5\%) & $85(82.5 \%)$ & 0.012 \\
\hline Mucinous adenocarcinoma $(n=2,1.5 \%)$ & $0(0 \%)$ & $2(100 \%)$ & \\
\hline Invasive lobular carcinoma $(n=3,2.3 \%)$ & I (33.3\%) & $2(66.7 \%)$ & \\
\hline Mixed ductal and lobular carcinoma $(n=3,2.3 \%)$ & $0(0 \%)$ & $3(100 \%)$ & \\
\hline Medullary carcinoma $(n=8,6.2 \%)$ & I (I2.5\%) & 7 (87.5\%) & \\
\hline Carcinoid $(n=2,1.5 \%)$ & $0(0 \%)$ & $2(100 \%)$ & \\
\hline Neuroendocrine carcinoma $(n=2,1.5 \%)$ & I (50\%) & I (50\%) & \\
\hline Invasive micropapillary carcinoma $(n=2,1.5 \%)$ & $0(0 \%)$ & $2(100 \%)$ & \\
\hline Apocrine carcinoma $(n=I, 0.8 \%)$ & $0(0 \%)$ & $\mathrm{I}(100 \%)$ & \\
\hline Normal $(n=4,3.1 \%)$ & $4(100 \%)$ & $0(0 \%)$ & \\
\hline \multicolumn{4}{|l|}{ Histological grade: } \\
\hline$I(n=3,2.4 \%)$ & $3(100 \%)$ & $0(0 \%)$ & 0.004 \\
\hline II $(n=63,50 \%)$ & $10(15.9 \%)$ & $53(84.1 \%)$ & \\
\hline III $(n=60,47.6 \%)$ & $8(13.3 \%)$ & $52(86.7 \%)$ & \\
\hline \multicolumn{4}{|l|}{ Histological stage: } \\
\hline$I(n=2,1.6 \%)$ & $2(100 \%)$ & $0(0 \%)$ & 0.001 \\
\hline II $(n=92,73 \%)$ & $18(19.6 \%)$ & $74(80.4 \%)$ & \\
\hline III $(n=32,25.4 \%)$ & I (3.1\%) & 31 (96.9\%) & \\
\hline \multicolumn{4}{|l|}{ Tumor size: } \\
\hline $\mathrm{TI}(\mathrm{n}=3,2.4 \%)$ & $2(66.7 \%)$ & $\mathrm{I}(33.3 \%)$ & 0.028 \\
\hline $\mathrm{T} 2(\mathrm{n}=74,58.7 \%)$ & $15(20.3 \%)$ & 59 (79.7\%) & \\
\hline T3 $(n=26,20.6 \%)$ & 3 (II.5\%) & $23(88.5 \%)$ & \\
\hline $\mathrm{T} 4(\mathrm{n}=23,18.3 \%)$ & I ( $4.3 \%)$ & $22(95.7 \%)$ & \\
\hline \multicolumn{4}{|l|}{ Lymph node metastasis: } \\
\hline Negative $(n=89,70.6 \%)$ & $16(18 \%)$ & $73(82 \%)$ & 0.372 \\
\hline Positive $(n=37,29.4 \%)$ & $5(13.5 \%)$ & $32(86.5 \%)$ & \\
\hline \multicolumn{4}{|l|}{ Distant metastasis: } \\
\hline Negative $(n=117,92.9 \%)$ & 21 (17.9\%) & $96(82.1 \%)$ & 0.921 \\
\hline Positive (n=9, 7.1\%) & $0(0 \%)$ & $9(100 \%)$ & \\
\hline \multicolumn{4}{|l|}{ PR status: } \\
\hline Negative $(n=123,97.6 \%)$ & 21 (17.1\%) & $102(82.9 \%)$ & 0.693 \\
\hline Positive $(n=3,2.4 \%)$ & $0(0 \%)$ & $3(100 \%)$ & \\
\hline \multicolumn{4}{|l|}{ ER status: } \\
\hline Negative $(n=122,96.8 \%)$ & $20(16.4 \%)$ & $102(83.6 \%)$ & 0.522 \\
\hline Positive $(n=4,3.2 \%)$ & I (25\%) & $3(75 \%)$ & \\
\hline \multicolumn{4}{|l|}{ HER2 status: } \\
\hline Negative $(n=123,97.6 \%)$ & $21(17.1 \%)$ & $102(82.9 \%)$ & 0.576 \\
\hline Positive ( $n=3,2.4 \%)$ & $0(0 \%)$ & $3(100 \%)$ & \\
\hline
\end{tabular}

grade I. Seventy-four patients $(58.7 \%)$ had tumor size of $2-3 \mathrm{~cm}$ (T2), while 26 patients $(20.6 \%)$ had tumor size larger than $5 \mathrm{~cm}$ (T3). Patients with tumors that metastasized to skin and chest wall (T4) were 23 in number (18.3\%), while only three patients $(2.4 \%)$ had tumor size less than $2 \mathrm{~cm}$ (T1). For ease of statistical comparison, the status of lymph node 
metastasis was also classified as lymph node-positive (29.4\%, 37 cases), or lymph node-negative (70.6\%, 89 cases). Pathological evaluation indicated that 9 patients (7.1\%) presented with distant metastasis, while 117 patients (92.9\%) were without distant metastasis. The expression levels for PR, ER and HER-2 were 2.4\% (three patients), $3.2 \%$ (four patients), and 3.2\% (four patients), respectively.

\section{CYP4ZI Expression and Its Relation to Clinicopathologic Characteristics}

The staining pattern and scoring guide for CYP4Z1 expression are presented in Figure 1. There was CYP4Z1 expression in 105 patients $(83.3 \%)$ where the immunoreactivity was distinctively found in the membrane or cytoplasm. All the four normal breast tissue samples had negative CYP4Z1 expression, as shown in Figure 2. The expression of CYP4Z1 was confirmed using appropriate negative and positive controls, and via inhibition of staining using CYP4Z1-blocking peptide. Results showed that no staining was seen in negative control, whereas strong staining was observed in breast cancer tissue (positive control). Moreover, there was no staining in TNBC tissue after incubation with a combination of CYP4Z1 antibody and blocking agent, as shown in Figure S1.
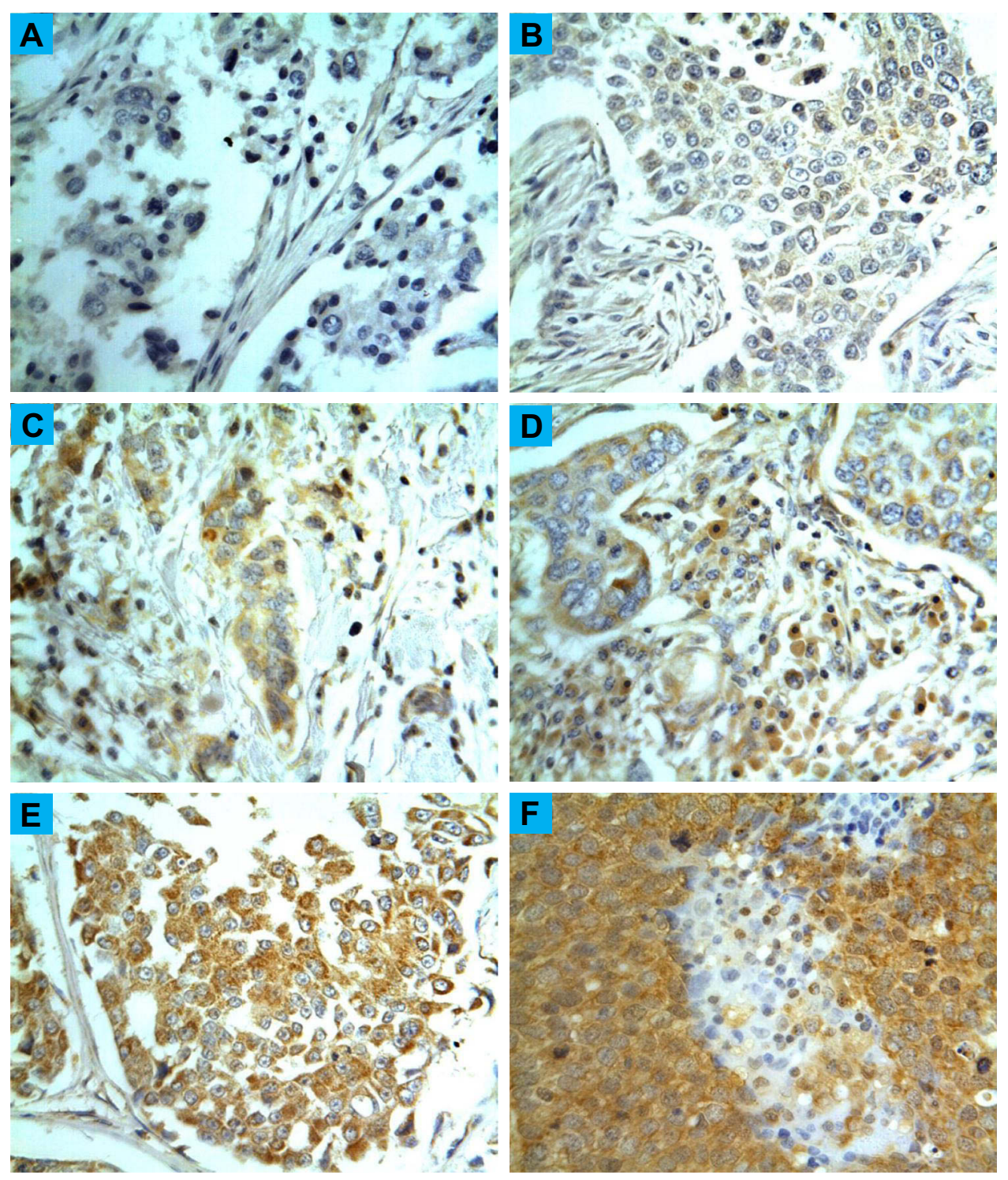

Figure I Staining pattern of CYP4Zlexpression and different scores of CYP4Zlexpression in TNBC. CYP4Zlexpression was displayed as clear membranous or cytoplasmic staining. (A) Score "0" showing no expression in the tissue at all. (B) Score "I" showing expression less than $1 \%$ of cells. (C) Score "2" showing expression between I-10\% of cells. (D) Score "3" showing expression between II-33\% of cells. (E) Score "4" showing expression in 34-66\% of the cells, (F) Score "5" showing expression in more than $67 \%$ of the cells. Magnification (X400). 

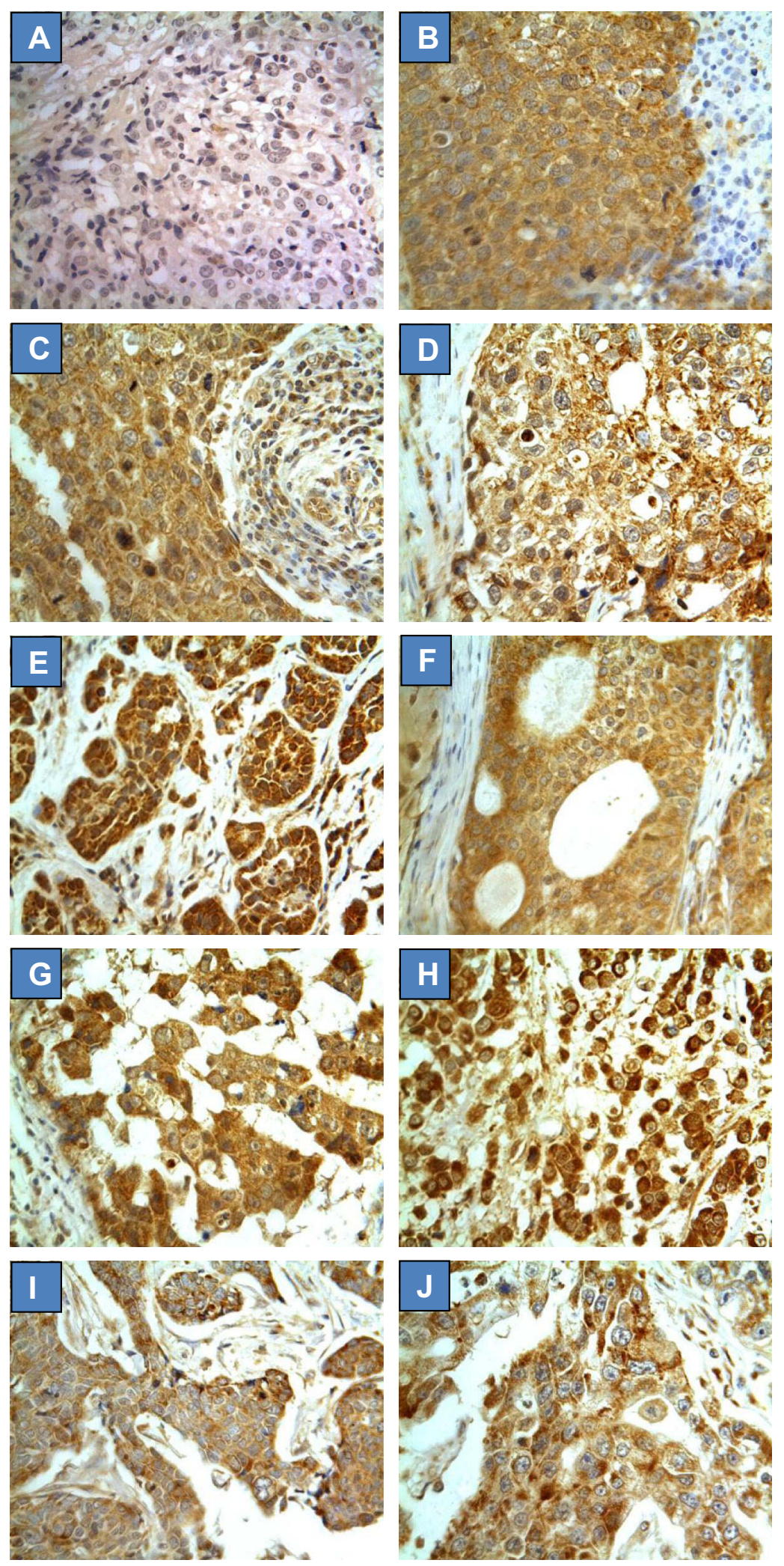

Figure $\mathbf{2}$ CYP4ZI expression in different types of TNBC. Tumours were classified on the basis of histological subtype. (A) Normal breast tissue, (B) invasive carcinoma of no special type, (C) medullary carcinoma, (D) invasive lobular carcinoma, (E) mixed ductal and lobular carcinoma, (F) mucinous adenocarcinoma, (G) invasive micropapillary carcinoma, (H) neuroendocrine carcinoma, (I) carcinoid and (J) apocrine carcinoma. Magnification (X400). 
The expression of CYP4Z1 had significant association with histopathology subtype, histology grade, histological stage and tumor size $(p<0.05$; Table 1$)$. The CYP4Z1 expression level varied markedly between normal specimens and different histopathology subtypes of TNBC. Moreover, CYP4Z1 had high expression in 103 patients (79.2\%) with invasive cancer of no special type. Almost all other histopathology subtypes expressed CYP4Z1. Moreover, there was a high level of CYP4Z1 expression in patients at grade III (86.7\%) and grade II (84.1\%), whereas patients with grade I showed complete negative expression. In addition, CYP4Z1 was highly expressed in patients at stage III (96.9\%) and stage II (80.4\%), when compared with complete negative expression in patients at stage I. Furthermore, patients who had high expression of CYP4Z1 had greater tumor size $(33.3 \%$ for $\mathrm{T} 1,79.7 \%$ for $\mathrm{T} 2,79.7 \%$ and $88.5 \%$ for $\mathrm{T} 3$, and $95.7 \%$ for $\mathrm{T} 4$ ). Conversely, there was no significant relationship between CYP4Z1 and age of the patients, status of lymph node metastasis, status of distant metastasis and expression levels of ER, PR and HER-2.

\section{The Association Between CYP4ZI and Prognosis in TNBC}

The major pathological subtype in this study (103 cases) was invasive carcinoma of no special type. Therefore, the cumulative survival and prognostic value of CYP4Z1 expression in this subtype of cases were analyzed. Patient survival data were evaluated with respect to CYP4Z1 expression groups using Kaplan-Meier curve and $\log$ rank test. Survival analysis showed poorer overall survival of TNBC patients characterized by high CYP4Z1 expression $(61.2 \%$; median $=41.3 \pm 1.9$ months $)$, relative to patients with low CYP4Z1 expression (72.2\%; median $=45.9 \pm 2.2$ months; $p=0.015)$. These results are shown in Figure 3. In addition, univariate Cox regression analysis revealed that CYP4Z1 expression, histopathological stage, tumor size, lymph node and distant metastasis had marked

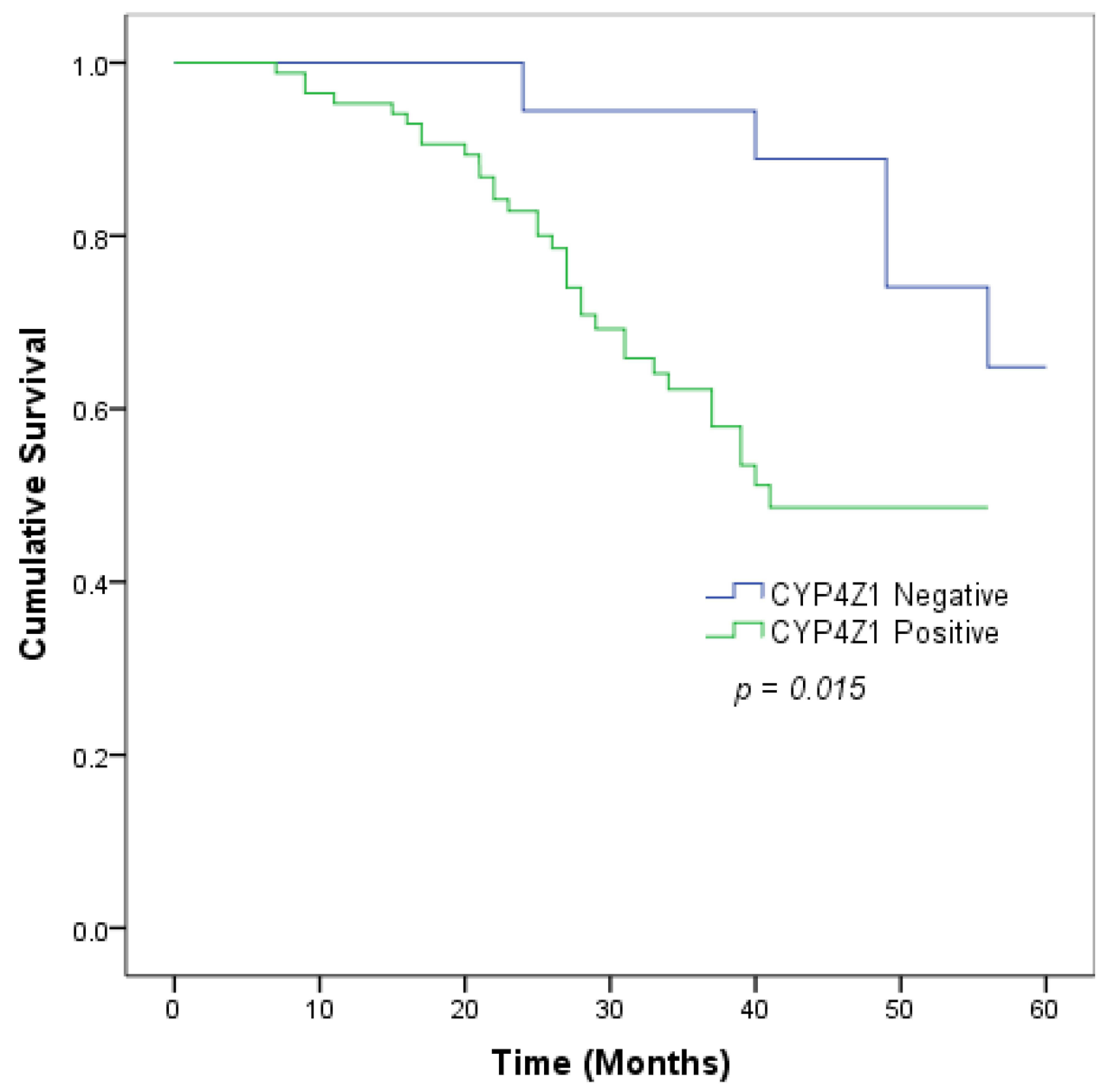

Figure 3 Kaplan-Meier curve showing overall survival of TNBC patients. 
Table 2 Univariate and Multivariate Analyses of Prognostic Variables

\begin{tabular}{|l|c|c|c|c|c|c|}
\hline \multirow{2}{*}{ Prognostic Variable } & \multicolumn{3}{|c|}{ Univariate } & \multicolumn{3}{c|}{ Multivariate } \\
\cline { 2 - 7 } & HR & $\mathbf{9 5 \% C l}$ & P-value & HR & 95\%Cl & P-value \\
\hline Age & 1.030 & $0.979-1.084$ & 0.252 & 1.021 & $0.971-1.073$ & 0.417 \\
Histological grade & 1.016 & $0.399-2.584$ & 0.974 & 0.617 & $0.320-1.189$ & 0.149 \\
Histological stage & 9.698 & $4.882-15.266$ & 0.001 & 11.10 & $3.189-16.687$ & 0.002 \\
Tumor size & 2.151 & $1.478-3.513$ & 0.008 & 0.653 & $0.365-1.167$ & 0.150 \\
Lymph node metastasis & 5.755 & $2.237-8.404$ & 0.002 & 1.373 & $0.677-3.368$ & 0.516 \\
Distant metastasis & 13.755 & $7.237-19.465$ & 0.001 & 9.112 & $2.456-17.799$ & 0.004 \\
CYP4ZI expression & 1.510 & $1.036-1.917$ & 0.031 & 1.320 & $1.137-1.996$ & 0.042 \\
\hline
\end{tabular}

correlation with overall survival ( $p<0.05$; Table 2). To further confirm and eliminate bias that may be caused by univariate analysis, multivariate Cox regression analysis was carried out. The results indicated that CYP4Z1 was identified as an independent prognostic predictor of poor overall survival of TNBC patients, among other factors, including histological stage and distant metastasis (Table 2).

\section{Discussion}

The global rise in mortality and morbidity in TNBC makes it a major health burden. This histopathological subtype of breast cancer is very aggressive and its prognosis is relatively poor, relative to other subclasses of breast carcinoma. The clinical management of TNBC is complicated and challenging due to the absence of targeted therapies. ${ }^{23}$ Therefore, there is a need for identification of new biomarkers and drug targets that can be effectively used in the management of TNBC. Several studies have indicated preferential expression of orphan CYP4 enzymes in certain types of tumors. ${ }^{7,24}$ One of these enzymes, CYP4Z1, has aroused great research interest. The selective expression of CYP4Z1 in breast cancers expressing various hormone receptors has been recently reported. ${ }^{8}$ Therefore, the present study was aimed at investigating CYP4Z1 expression in breast cancer cells lacking the hormone receptor expressions of ER, PR and HER-2.

This is the first study to define CYP4Z1 expression in TNBC. The results showed that CYP4Z1 was highly expressed in $83.3 \%$ of TNBC patients, in contrast to negative CYP4ZI expression in normal tissues. This disparity in CYP4Z1 expression between breast cancers and healthy breast tissues has been reported in several studies. ${ }^{3,7,8}$ Interestingly, serum CYP4Z1 autoantibodies are generated due to the translocation of CYP4Z1 expression to the cell surface of breast cancers. ${ }^{13}$ These serum autoantibodies against CYP4Z1 may act as a diagnostic indexes for mammary cancer. It is crucial to note that CYP4Z1 expression was associated with poor survival of TNBC patients, and it appeared to be an independent prognostic predictor of overall survival. This is a novel finding, and it is in agreement with earlier findings where differential CYP4Z1 expression was identified as a prognostic marker for ovarian, prostate and cervical cancers. $5,6,11$

Based on the fact that aggressive TNBC expressed significantly high level of CYP4Z1, the CYP4Z1 expression was associated with tumor histopathological subtypes. This is in agreement with earlier findings showing similar pattern of expression amongst different histopathological subtypes of breast cancers. ${ }^{7,8}$ The present study also showed that there were significant differences in CYP4Z1 expression in relation to histological grade, histological stage and tumor size. These results are consistent with the results obtained in other studies on the role of CYP4Z1 as a clinicopathological marker in different tumors, particularly breast cancer. For instance, CYP4Z1 expression was markedly correlated with histological grade and histological stage: CYP4Z1 level was elevated in advanced-stage patients with high grades in histopathology. ${ }^{3,8}$ These observations provide insight into the molecular mechanisms through which CYP4Z1 acts. Overexpression of CYP4Z1 enhanced cellular proliferation, tumor angiogenesis, and the spread of breast tumor cells. ${ }^{10,25}$ Moreover, CYP4Z1 expression inhibited apoptosis and induced stemness and tamoxifen insensitivity in breast carcinoma. ${ }^{26-28}$ Overall, CYP4Z1 enzyme appears to play a vital role in breast malignancy, but its molecular mechanisms are yet to be fully elucidated.

Although the unique expression of CYP4Z1 marks it as a promising molecule for directed delivery of chemotherapeutics, the development of CYP4Z1 targeting therapies faces many challenges. One of the key challenges is 
related to the limited knowledge about the catalytic properties of CYP4Z1. Several studies have investigated the functional properties of CYP4Z1 and attempted to identify possible substrate candidates. ${ }^{10,14,15,29}$ The CYP4Z1 enzyme is recognized as a fatty acid hydroxylase. The first two fatty acid substrates for CYP4Z1 are lauric acid and myristic acid. These mid-chain fatty acids were metabolized to different $\omega$-mono-hydroxylated products, while short and long-chain saturated fatty acids, as well as unsaturated fatty acids, were not metabolized. ${ }^{14}$ In a similar pattern of reaction, CYP4Z1 catalyzed the metabolism of arachidonic acid to 20-HETE, while eicosadienoic acid was metabolized to an unknown product. These conversions were suppressed by blocking CYP4Z1 enzyme activity with the known CYP4 inhibitor, ie, HET0016. ${ }^{10}$ The findings in this study are somewhat at odds with those of a recent study, which reported the absence of 20-HETE metabolites, and the preferential generation of 14.15EET. $^{15}$ Recently, the first luminogenic substrate for CYP4Z1 (luciferin benzyl ether) was identified by screening a set of luminogenic substrates using permeabilized cells of fission yeast expressing CYP4Z1. ${ }^{29}$ Overall, these studies have aided the development of the first selective mechanism-reliant suppressor, 8-[( $1 H$-benzotriazol-1-yl $)$ amino] octanoate. This inhibitor efficiently blocks the production of 14.15-EET in CYP4Z1-positive breast carcinoma cells, in contrast to weak inhibitory profiles against other CYPs. ${ }^{30}$ These recent advances are useful for the molecular probing of the functional activities of CYP4Z1 and its involvement in breast cancer.

\section{Conclusion}

This is the first investigation that identified a unique expression of CYP4Z1 in all histopathological subtypes of TNBC, in contrast to almost no expression in the corresponding normal breast tissues. There was a significantly higher frequency of CYP4Z1 expression in patients with high grades in histopathology and those at advanced stages of disease. Moreover, there was a strong differential in CYP4Z1 expression amongst all tumor sizes. Interestingly, CYP4Z1 expression was pinpointed as an independent factor for poor survival of TNBC patients. Therefore, CYP4Z1 may serve as a potential target and vital biomarker for TNBC.

\section{Acknowledgments}

The authors are very thankful to all the associated personnel for any reference who contributed towards the purpose of this research. Moreover, a special thanks to the
Deanship of Scientific Research at Mut'ah University. Our sincere thanks are due to Dr. Ahmed Adnan Alabadleh for his kind support and work.

\section{Author Contributions}

All authors made a significant contribution to the work reported, whether that is in the conception, study design, execution, acquisition of data, analysis and interpretation, or in all these areas; took part in drafting, revising or critically reviewing the article; gave final approval of the version to be published; have agreed on the journal to which the article has been submitted; and agree to be accountable for all aspects of the work.

\section{Disclosure}

The authors report no conflicts of interest in this work.

\section{References}

1. Cocco S, Piezzo M, Calabrese A, et al. Biomarkers in triple-negative breast cancer: state-of-The-art and future perspectives. Int J Mol Sci. 2020;21(13):4579. doi:10.3390/ijms21134579

2. Siegel RL, Miller KD, Jemal A. Cancer statistics, 2020. CA Cancer J Clin. 2020;70(1):7-30. doi:10.3322/caac.21590

3. Rieger MA, Ebner R, Bell DR, et al. Identification of a novel mammary-restricted cytochrome p450, cyp4z1, with overexpression in breast carcinoma. Cancer Res. 2004;64(7):2357-2364. doi:10. 1158/0008-5472.can-03-0849

4. Yang X, Hutter M, Goh WWB, Bureik M. Cyp4z1 - a human cytochrome p450 enzyme that might hold the key to curing breast cancer. Curr Pharm Des. 2017;23(14):2060-2064. doi:10.2174/1381612823 666170207150156

5. Tradonsky A, Rubin T, Beck R, Ring B, Seitz R, Mair S. A search for reliable molecular markers of prognosis in prostate cancer: a study of 240 cases. Am J Clin Pathol. 2012;137(6):918-930. doi:10.1309/ AJCPF3QWIG8FWXIH

6. Downie D, McFadyen MC, Rooney PH, et al. Profiling cytochrome p450 expression in ovarian cancer: identification of prognostic markers. Clin Cancer Res. 2005;11(20):7369-7375. doi:10.1158/ 1078-0432.CCR-05-0466

7. Murray GI, Patimalla S, Stewart KN, Miller ID, Heys SD. Profiling the expression of cytochrome p450 in breast cancer. Histopathology. 2009;57(2):202-211. doi:10.1111/j.1365-2559.2010.03606.x

8. Al-Saraireh YM, Alboaisa NS, Alrawashdeh HM, et al. Screening of cytochrome 4z1 expression in human non-neoplastic, pre-neoplastic and neoplastic tissues. Ecancermedicalscience. 2020;14:1114. doi:10. 3332/ecancer.2020.1114

9. Al-Saraireh YM, Alshammari FO, Youssef AM, et al. Profiling of cyp4z1 and cyp1b1 expression in bladder cancers. Sci Rep. 2021;11 (1):1-8. doi:10.1038/s41598-021-85188-4

10. Yu W, Chai H, Li Y, et al. Increased expression of cyp4z1 promotes tumor angiogenesis and growth in human breast cancer. Toxicol Appl Pharmacol. 2012;264(1):73-83. doi:10.1016/j.taap.2012.07.019

11. Al-saraireh YM, Alshammari FOFO, Youssef AMM, et al. Cytochrome $4 \mathrm{z} 1$ expression is correlated with poor prognosis in patients with cervical cancer. Curr Oncol. 2021;28(5):3573-3584.

12. Khayeka-Wandabwa $\mathrm{C}$, Ma X, Cao X, et al. Plasma membrane localization of cyp4z1 and cyp19a1 and the detection of anti-cyp19a1 autoantibodies in humans. Int Immunopharmacol. 2019;73:64-71. doi:10.1016/j.intimp.2019.05.003 
13. Nunna V, Jalal N, Bureik M. Anti-cyp4z1 autoantibodies detected in breast cancer patients. Cell Mol Immunol. 2017;14(6):572-574. doi:10.1038/cmi.2017.21.

14. Zollner A, Dragan CA, Pistorius D, et al. Human cyp4z1 catalyzes the in-chain hydroxylation of lauric acid and myristic acid. Biol Chem. 2009;390(4):313-317. doi:10.1515/BC.2009.030

15. McDonald MG, Ray S, Amorosi CJ, et al. Expression and functional characterization of breast cancer-associated cytochrome p450 4z1 in saccharomyces cerevisiae. Drug Metab Dispos. 2017;45 (12):1364-1371. doi:10.1124/dmd.117.078188

16. Panigrahy D, Greene ER, Pozzi A, Wang DW, Zeldin DC. Eet signaling in cancer. Cancer Metastasis Rev. 2011;30(3-4):525-540. doi:10.1007/s10555-011-9315-y

17. Luo J, Feng -X-X, Luo C, et al. 14,15-eet induces the infiltration and tumor-promoting function of neutrophils to trigger the growth of minimal dormant metastases. Oncotarget. 2016;7(28):43324-43336. doi:10.18632/oncotarget.9709

18. Bruno RD, Njar VCO. Targeting cytochrome p450 enzymes: a new approach in anti-cancer drug development. Bioorg Med Chem. 2007;15(15):5047-5060. doi:10.1016/j.bmc.2007.05.046

19. Fitzgibbons PL, Dillon DA, Alsabeh R, et al. Template for reporting results of biomarker testing of specimens from patients with carcinoma of the breast. Arch Pathol Lab Med. 2014;138(5):595-601. doi:10.5858/arpa.2013-0566-CP

20. Harvey JM, Clark GM, Osborne CK, Allred DC. Estrogen receptor status by immunohistochemistry is superior to the ligand-binding assay for predicting response to adjuvant endocrine therapy in breast cancer. J Clin Oncol. 1999;17(5):1474-1481. doi:10.1200/ jco.1999.17.5.1474

21. Daltoé RD, Madeira KP, de Carvalho AA, de Rezende LC, Silva IV, Rangel LB. Evaluation of the progesterone receptor status in breast cancer using three different antibodies: a comparison by Allred score system. Int J Clin Exp Pathol. 2014;7(1):331-339.
22. Allred DC. Problems and solutions in the evaluation of hormone receptors in breast cancer. J Clin Oncol. 2008;26(15):2433-2435. doi:10.1200/jco.2007.15.7800

23. Anders C, Carey LA. Understanding and treating triple-negative breast cancer. Oncology. 2008;22(11):1233-1239; discussion 1239-1240, 1243.

24. Alexanian A, Miller B, Roman RJ, Sorokin A. 20-hete-producing enzymes are up-regulated in human cancers. Cancer Genom Proteom. 2012;9(4):163-169.

25. Zheng L, Li X, Gu Y, Lv X, Xi T. The 3'utr of the pseudogene cyp $4 z 2 p$ promotes tumor angiogenesis in breast cancer by acting as a cerna for cyp4z1. Breast Cancer Res Treat. 2015;150(1):105-118. doi:10.1007/s10549-015-3298-2

26. Zheng L, Li X, Meng X, et al. Competing endogenous RNA networks of cyp4z1 and pseudogene cyp4z2p confer tamoxifen resistance in breast cancer. Mol Cell Endocrinol. 2016;427:133-142. doi:10.1016/j.mce.2016.03.012.

27. Li C, Zheng L, Xin Y, et al. The competing endogenous RNA network of cyp4z1 and pseudogene cyp4z2p exerts an anti-apoptotic function in breast cancer. FEBS Lett. 2017;591 (7):991-1000. doi:10.1002/1873-3468.12608

28. Zheng L, Guo Q, Xiang C, et al. Transcriptional factor six 2 promotes the competitive endogenous RNA network between cyp4z1 and pseudogene cyp4z2p responsible for maintaining the stemness of breast cancer cells. J Hematol Oncol. 2019;12(1):23. doi:10.1186/ s13045-019-0697-6

29. Yan Q, Machalz D, Zollner A, Sorensen EJ, Wolber G, Bureik M. Efficient substrate screening and inhibitor testing of human cyp4z1 using permeabilized recombinant fission yeast. Biochem Pharmacol. 2017;146:174-187. doi:10.1016/j.bcp.2017.09.011

30. Kowalski JP, McDonald MG, Pelletier RD, Hanenberg H, Wiek C, Rettie AE. Design and characterization of the first selective and potent mechanism-based inhibitor of cytochrome p450 4z1. J Med Chem. 2020;63(9):4824-4836. doi:10.1021/acs.jmedchem.0c00101
Breast Cancer: Targets and Therapy

\section{Publish your work in this journal}

Breast Cancer - Targets and Therapy is an international, peer-reviewed open access journal focusing on breast cancer research, identification of therapeutic targets and the optimal use of preventative and integrated treatment interventions to achieve improved outcomes, enhanced survival and quality of life for the cancer patient.

\section{Dovepress}

The manuscript management system is completely online and includes a very quick and fair peer-review system, which is all easy to use. Visit http://www.dovepress.com/testimonials.php to read real quotes from published authors. 